சங்க மற்றும் அறநெறிக்கால பண்டைத் தமிழர்களின் வாழ்வில் கலைகள் -சிற்பக்கலை மற்றும் ஓவியக்கலையை அடிப்படையாகக் கொண்ட ஆய்வு கி. சர்வேஸ்வரன் அ, *

भ இந்து நாகரிகத்துறை, கிழக்குப் பல்கலைக்கழகம், வந்தாறுமூலை, இலங்கை.

\title{
Arts in the Life of Sangam and Moral Ancient Tamils - A Research Based on Sculpture and Painting
}

\author{
K. Sarveshwaran ${ }^{a}$, \\ a Department of Hindu Civilization, Eastern University, Vantharumoolai, Sri Lanka.
}

* Corresponding Author: sarwesh1177@gmail.com

Received: 13-03-2021

Revised: 07-12-2021

Accepted: 16-12-2021

Published: 11-02-2022

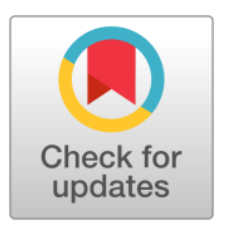

\begin{abstract}
Literature can be said to be an activity or replica phenomenon that reveals a kind of attention. Tamil literature, which has a very long history, has a wide variety of concepts. The specialty of literature is to make these ideas world-renowned in a way that reflects a community. It must be said that these explanation0s did not hesitate to bear the various elements of the ancient Tamils in that way. Within those elements the arts are remarkable. Thus the arts are unique in the ancient Tamil cultural tradition. The study is being conducted under the title Arts in the life of Sangam and Moral ancient Tamils A Research Based on Sculpture and Painting', realizing the immense creative arts in human energy. The study was carried out to study the literature on sculpture and painting, and to explore the true nature of the art of those who still preserve the art and preserve it. The main sources of this study are literature related to sculpture and painting. Through this study, the arts could be identified as an excellent tool for the development of anthropology, and a complete understanding of the arts in the case of the ancient Tamils could be obtained.
\end{abstract}

Keywords: Art, Painting, Sculpture, Image, Puppet, Ancient, Ancient Tamil.

\section{முன்னுரை}

கலையென்பது 'கலா' என்ற வினையடியிலிருந்து தோன்றியது என்பர் கலா என்பது சதா வளர்த்தல் எனப் பொருள்படும். அவ்வாறாக நாம் வளர்த்து வந்த கலைகள் பல காணப்படுகின்றன. அவையாவன சிற்பக்கலை, கட்டிடக் கலை, ஓவியக் கலை, நாட்டியக் கலை, நாடக் கலை, இலைக் கலை என்று ஆய கலைகள் அறுபத்து நான்காகும். இவையனைத்திற்கும் ஆதாரமாகக் திகழ்வது நமது பண்பாடும், மதப்பின்னணியுமேயாகும். மனிதம் கண்டறிந்த மனமகிழ்விற்கான வழிகளில் கலையும் ஒன்றாகும். அவ்வாறு நம் மதம் சார்ந்த இவ் ஆயகலைகள் அறுபத்துநான்கும். உலகி பலபிரதேசங்களிலும் பல்வேறு மொழி பேசுபவர்களிடமும் வழக்கத்திலுள்ள. 9வ்வாறு வழக்கத்திலுள்ளவைக்குமென சில மொழிரீதியான, மற்றும் தத்தம் பண்பாடு ரீதியாக ேேறுபாடுகள் காணப்படுகின்றன. அவ்வாறான மாறுபாடே தமிழகக் கலைகளிலும் மிளிர்கின்றது.

உலக மாந்தர்கள் யாவர்க்கும் பொதுவான கலைகள் தத்தம் மதம் சாரும்போதும், பண்பாடு சாரும் போதும் பல மாற்றங்களை தனதாக்கி வளர்வு காணும். இது இயல்பாகும். இவ் இயல்புக்கு 
தமிழர் கலைகளும் உட்பட்வை. சுமார் இரண்டாயிரம் ஆண்டுகளுக்கு முன்பிருந்தே தமிழர்கள் வளர்த்த கலைகள் ஏராளம், அவற்றில் தமிழர் உணர்வுகளும், சிந்தனைகளும் புரையோடிப்போயுள்ளன என்பது மறுக்கமுடியாத உண்மையாகும். எனவே எம்மவர் கலை குறித்த சிந்தனைகளை அறிய எமக்கு வரலாற்று ஆதாரமாக எஞ்சியுள்ளவை பண்டைத் தமிழர்களின் பதினெண்கீழ்க் கணக்கு நூல்களும், பதினெண் மற்கணக்கு நூல்களும், அறநெறிக்கால நூல்களுமேயாகும். அவற்றில் எஞ்சியுள்ள நூல்களின் வாயிலாக நாம் பண்டைத் தமிழர்களின் வாழ்வியற் கோலங்களை அறிந்துகொள்கின்றோம். அதன்படி பண்டைத் தமிழர்களின் வாழ்வியற் கோலங்களில் ஒன்றான கலைவெளிப்பாடு பற்றியும், அக் கலைகளில் ஓவிய மற்றும் சிற்ப கலை பற்றியும் இவ் இலக்கியங்களை ஆதாரமாக்கியே ஆராயமுற்படுவோம்.

\section{சங்க மற்றும் அறநெறிக்கால இ இலக்கியங்களில் ஓவியக் கலை பற்றிய குறிப்புக்கள்.}

ஓவு, ஓவம் என்பன ஓவியம் என்ற பொருளுடையன. இச் சொற்கள் சங்க இலக்கியத்தில் பெருவழக்கத்திலுள்ளன. ஒப்பு உவமை என்ற அடிப்படையில் ஒன்றன் வடிவை அவ்வாறே வரைதல் பற்றிய பொருண்மையில் இச்சொற்கள் தோன்றின எனலாம். ஓவுறழ், நெடுஞ்சுவர் என்ற இடத்து 'ஓ' என்ற ஓரெழுத்து மொழியே ஓவியத்தைக் குறித்தது. பழைய கற்காலத்திலிருந்தே ஓவியக்கலை வரலாறு தோன்றிவிட்டது. 30,000 ஆண்டுகளுக்கு முன்னால் பனிக்கட்டிகளுடனே வாழ்ந்த ஐரோப்பிய மனிதன் எலும்புகள் மீதும் தந்தங்களின் மீதும் விலங்குகளின் உருவங்களைச் செதுக்கினான் என அறிய முடிகின்றது. இவ்வாறாகவே ஓவியக்கலை தோற்றம் பெற்றிருந்தது. மனிதனின் சிந்தனைகளையும் தான் கண்ட அழகியல் அம்சங்களையும் தம்முடைய வீர சாகசங்களையும் மீண்டும் மீண்டும் நினைவுகூர்ந்து மகிழ்ச்சியடைவதற்காக அவற்றையெல்லாம் ஓவியங்களாக வரைந்து வைத்து ரசித்தான் ஆதிகால மனிதன்.

இதனடிப்படையில் தோன்றிய ஓவியக்கலை உலகின் அனைத்து மனிதவர்கத்திற்கும் அனைத்து நாகரிகத்தினருக்கும் சொந்தமானதும் பொதுமையானதுமாகும். ஆதிகாலத்திலேயே தோன்றிய நாகரிகங்கள் அனைத்திலும் இன்றும் ஓவியக்கலையின் எச்சசொச்சங்கள் அவ்வாறே மிகுதியாக காட்சியளிக்கின்ற. அவை அவர்களின் கலைப் பண்பாட்டையும் திறனையும் வெளிக்காட்டுகின்றனவையாக மட்டுமன்றி அவர்களின் வாழ்க்கை வரலாற்றின் கண்ணாடியாகவும் உள்ளன.

கற்கால மனிதன் தான் வாழ்ந்த குகைகளில் காட்டெருமை, மான் ஆகிய வேட்டைக்குரிய விலங்குகளை வரைந்திருந்தான். பிரான்ஸ் நாட்டில் உள்ள குகைகளில் இவ் ஓவியங்கள் கண்டு பிடிக்கப்பட்டன. ஸ்பெயின் நாட்டு ஆதி மனிதன் வண்ணந் தீட்டப்பட்ட விலங்குருவங்களை வரைந்திருந்தான். அதேபோல மத்திய இந்தியாவின் குகைகளில் செம்மண் தீட்டப்பட்ட வேட்டையாடும் ஓவியங்கள் காணப்படுகின்றன. காண்டாமிருகம், ஒட்டகச்சிவிங்கி, கலைமான் போன்றனவற்றை ஆதி மனிதன் வேட்டையாடும் காட்சிகள் அவற்றில் சிறப்பானவையாகும். அத்துடன் சிந்துவெளி நாகரிக காலத்திலும் சிறப்பான மட்பாண்ட ஓவியங்கள் வரையப்பட்டிருந்தமையும் குறிப்பிடத்தக்கது (Pararajasingham, 2017). இவ்வாறாக பல நாடுகள் தாண்டி, நாகரிகங்கள் தாண்டி, பலபண்பாடுகள் தாண்டி, வளரப்பெற்ற ஓவியக்கலை ஆதிகாலத் தமிழர்களையும் பாதிக்கத் தவறவில்லை. பண்டைத் தமிழ் மக்களின் அழகுபெறு ஓவியங்கள் இன்றும் பல்வேறு இடங்களில் காணக்கிடைக்கின்றன. அவ்வாறான இடங்களாக,

- கீழ்வாலை (தென்னார்க்காடு)

• செத்தவரை (தென்னார்க்காடு)

• முத்துப்பட்டி (மதுரை)

• அணைப்பட்டி (மதுரை) 
• திருமலை (மதுரை)

• காமயகவுண்டன்பட்டி (மதுரை)

• வேட்டைக்காரன் பாளையம் (கோவை)

போன்ற இடங்கள் அடையாளம் காணப்பட்டுள்ளன. இவ் இடங்களிலெல்லாம் இன்றும் பல பாறை ஓவியங்கள் காணப்படுகின்றன. எனப் பவுன்துரை குறிப்பிடுகின்றார். இப்பாறை ஓவியங்களில் பெரும்பாலானவை வேட்டைக்கால ஊழியைக் காட்டுவனவாகும். உணவுப் பொருட்களைத் திரட்டும் காலத்திற்கு முற்பட்ட வேட்டைக்கால வாழ்வு இங்கே சித்திரிக்கப்படுகின்றது. ஆனால் சிந்துவெளி ஓவியங்களுக்கும் இத் தமிழகப் பாறை ஓவியங்களுக்குமிடையே குறிப்பிடத்தக்க வேறுபாடுகள் காணப்படுவதும் தெரிய வருகின்றது. இதற்குக் காரணமாக இரு காலங்களுக்குமிடையிலான பேரிடைவெளியை வரலாற்று ஆசிரியர்கள் குறிப்பிடுகின்றனர் (Navarathinam,2006).

அதனடிப்படையில் நோக்கின், சிந்துவெளி ஓவியங்கள் அம்மனிதர்களின் பொழுதுபோக்கு மற்றும் கலை உணர்வினைக் காட்டுவதாகின்றன. சிந்துவெளிக்குப் பின் ஓவியக்கலையை இந்தியாவில் வளர்த்துச் சென்ற பெருமை பௌத்த சமயத்தவர்களையே சாருகின்றது. இதற்கு தக்க உதாரணம் இந்தியாவில் கௌதம புத்தரின் உருவம் இன்றும் ஓர் வரைபொருளாக பெரு மதிப்புப் பெறுகின்றமையேயாகும். சங்க கால ஓவியங்கள் பலநூற்றாண்டுகளின் கலைவளர் தன்மைகமை வெளிப்படுத்துகின்றன. ஆரம்பத்தில் விலங்கு பறவை, ஆகியவற்றை உருத்தீட்டிய காலம் ஆகின்றது. அதன் பின்னர் மனித உருவங்களை தீட்டத் துவங்கினர். மனித செயற்பாடுகள் பலவும் ஓவியங்களாகின. சங்க காலத்திலே ஓவியக்கலை இரண்டு வகையாக வரையப்பட்டிருந்தது.

1. இயற்கையை ஓவியமாக வரைதல்,

2. புராதனமான பழங்கதைகளை ஓவியம் செய்தல்,

எனும் இரண்டையும் அடியொற்றியதாகவே சங்ககால ஓவியங்கள் காணப்பட்டுள்ளன. என அறியலாம். இது பற்றி பேராசிரியர் வைத்தியலிங்கம் அவர்கள் குறிப்பிடுகையில்,

'பழந்தமிழர்கள் இயற்கையைத் துய்த்தனர். இயற்கையில் தாம் துய்த்த அழகிய அனுபவத்தை ஓவியமாகப் பதிவு செய்வதில் இன்பமெய்தினர். மனிதன் ஒருவன் வானத்தில் செடிகொடிகளில் பறவைகளில் விலங்குகளில் கவர்ச்சிமிக்க பல வண்ணங்களையும், உருவங்களையும் கண்டபோது அவற்றின் அழகால் அவன் ஈர்க்கப்பட்டான். அவன் ஆழ நினைத்தான். அவன் உளநிலையும் போலச் செய்யும் கருத்து நிலையும் இயற்கையில் பெறும் அழகியல் அனுபவத்தை வரைகலையாகவும் ஓவியமாகவும் காணவிழைந்தது.' என்கின்றார் (Vaithilingam, 1996). இவரது கருத்திற்கு வழிகோலியதாக மூலாதாரமாக நெடுநல்வாடையானது விளங்குகின்றது எனலாம். அந்நெடுநல்வாடையிலே,

'வெள்ளி யன்ன விளக்குஞ் சுதையுரீஇ

முணிகண் டன்ன மாத்திரட் டினண்காழ்ச்

செம்பியன்றன்ன செய்வுறு நெடுஞ்சுவர்

உருவப் பல்பூ ஒரு கொடி வளை இக்

கருவொடு பெயரிய காண்பின் நல்லில்

எனவரும் பாடலானது சாந்து கொண்டு சமைத்துப் பூசிய இல்லத்துச் சுவர்களில் வல்லி சாதியாகி ஒப்பில்லாத கொடியை யெழுதி அழகுபடுத்தியமை பற்றிக் குறிப்பிடுகின்றது. நீர் நிலைகளை அழகோவியமாக வரைந்தமை பற்றியே சிறுபாணாற்றுப்படை குறிப்பிடுகின்றது.

'மலரும் தாதும் உதிர்ந்த நீர்த்துறையின் அழகைப் பண்டு ஓவியமாக்கினர்.' எனும் குறிப்பும் காணப்படுகின்றது.

அதேபோல பல்வேறு வண்ணக்கலவைகள் விளையாடிய ஓவியக் கிழியென வெறிக்களம் தோற்றுதல் பற்றி, 
'நீலத்தன்ன பைம்பயிர் மிசைதொறும்

வெள்ளி யன்ன ஓள்வீ யுதிர்ந்து

கரிமுகிழ் முசுண்டையொடு முல்லை தாஅய்

மணிமருள் நெய்தல் உறழக் காமர்

துணிநீர் மெல்லவல் தொய்யிலொடு மலர

வல்லோன் தைஇய வெறிக்களங் கடுப்ப'

(மதுரைக்காஞ்சி 279ம் பாடல்)

என்ற மதுரைக்காஞ்சி குறிப்பிடுகின்றது. ஓர் வாவியில் பல வகை மலர்களும், கொடிகளும் ஓன்றாக பிணங்கி காணப்படும் காட்சி ஓவியமாக வரையப்பட்டது பற்றியே இப்பாடல் குறிப்பிடுகின்றது. மதுரையில் நாளங்காடியையும், அல்லங்காடியையும் காணும் காட்சி ஓவியம் கண்டதைப் போன்றிருந்தது என ஓரிடத்தை உவமானமாக காட்ட விழையும் போதே புலவர் ஓவியம் பற்றிக் குறிப்பிடுகின்றார்.



என்ற வரி, சித்திரத்தைப் போல வேலைப்பாடுகள் அமைந்த நல்ல வீடு என்று வீட்டினைக் கூற விழையும்போது உவமானமாக சித்திரம் பொருத்திக் கூறப்படுகின்றது (Sithambaranar, 2004). மேலும் சங்கப் பாடல்களில் ஓவியம் பெரிதும் ஒப்பீட்டிற்கும், உவமானத்திற்கும் மேலும் பல இடங்களில் பயன்படுத்தப்பட்டுள்ளதை காணலாம். உதாரணமாக,

'ஓவத் தன்ன உருகெழு நெடுநகர்'

எனத்தொடரும் பதிற்றுப் பத்தின் பெருங்குன்றூர்க் கிழாரின் பாடலில் ஓவியம் போன்றதான அரண்மனை எனும் வர்ணனை காட்டப்படுகின்றமையைக் கூறலாம். அரசர்கள் நாட்காலையில் வழிபடுந் தெய்வம் சித்திரகாரிகளால் தீட்டப்பெற்றிருந்தது என்பதனையும் மதுரைக்காஞ்சி குறிப்பிடுகின்றது. அத்துடன் அரசர்களின் வளமனைகளில் உறங்குங் கட்டிலின் மேலிடத்தே புணர்ச்சி விருப்பத்திற்குரிய ஓவியம் வரையப்பட்டிருந்ததாக நெடுநல்வாடை குறிப்பிடுகின்றது.

'புதுவ தியன்ற மெழுகு செய் படமிசைத்

திண்ணிலை மருப்பின் ஆடுதலை யாக

விண்ணூர்பு திரிதரும் வீங்கு செலன் மண்டிலத்து

முரண்மிகு சிறப்பிற் செல்வனோடு நிலைஇய

ஊரோகிணி நினைவனன் நோக்கி நெடிதுயிரா'

வருந்தினள் தேவியென்பார் (Sithambaranar, 2004). புலவர். சந்திரனும் (திங்களும்) உரோகிணியும், மானிட வடிவில் ஓவியமாக வரையப்பட்டுள்ளமை பற்றியே புராணக் கதையை அடியொற்றியதான இவ் ஓவியத்தின் சிறப்புப் பற்றி மேற்கண்ட பாடல் சிறப்பித்துச் செல்கின்றது. அஷ்மாசனகிரி எனப்படும் மறற்குதியில் 12 இராசிகளும் வரையப்பட்டிருந்ததாகவும், சந்திரனது உருவமும் உரோகிணியின் உருவமும் பிரியாது நிற்கும் நிலையில் வரையப்பட்டிருப்பதாகவும், பரிபாடல் குறிப்பிடுகின்றது,

'திண்நிலை மருப்பின் ஆடு தலையாக

விண்ஊர்பு திரிதரும் வீங்குசெலல் மண்டிலத்து

முரண்மிகு சிறப்பின் செல்வனோடு நிலைஇய

உரோகிணி' 
என்றவாறு அப்பாடல் வரிகள் அமைந்து காணப்படுகின்றமை பற்றி சாமி சிதம்பரனார் குறிப்பிடுகின்றார் (Sithambaranar, 2004). இக்கூற்று மேல் நிலை விதானங்களில் அழகிய ஓவியங்கள் வரையப்பட்டிருந்தமைக்கும் மேலும் சிறந்த உதாரணமாகும்.

\section{பண்டைத் தமிழிலக்கியங்களில் வண்ணம் பற்றிய சிந்தனைகள்.}

நெடுநல்வாடை ஓவியம் பற்றிய ஏராளமான மேற்கோள்களை முன்வைக்கின்றது. அத்துடன் ஓவியத்திற்கு தேவையான வண்ணம் பற்றியும் எடுத்துரைக்கின்றது. 'புனையாஓவியம்' என்ற தொடர் ஓவியப் படிநிலையை விளக்குவதாக பாடல்களில் இடம்பெற்றுள்து. நச்சினார்க்கினியர் 'வண்ணங்களைக் கொண்டெழுதாத வடிவைக் கோட்டின சித்திரத்தை ொப்ப....' என உரையெழுதுகின்றார். இப்பகுதியில் பெண்ணொருத்தியின் மேனி வடிவினையும் நிறத்தினையும் ஓவியக் கலைப் பாங்கில் வெளிப்படுத்துவது எண்ணுவதற்குரியதாகின்றது.

'அம்மா சூர்ந்த அவிர்நூற் கலிங்கமொடு

புனையா ஓவியங் கடுப்பப் புனைவில்

தளிரேர் மேனித் தாய கணங்கின்'

மேற்கண்ட இப்பாடலின் வரிகளினால் வண்ணந் தீட்டுவதனலாலேயே ஓரோவியம் புனைவு பெறும் எனும் கருத்தினை நெடுநல்வாடை குறிப்பிடுவதனைக் காணலாம். வண்ணங்களைக் குறித்த தெளிவான அறிவு பண்டைத் தமிழர்களிடம் அதிகமாகவே இருந்துள்ளது என்றே கூறவேண்டும்.

- அழலுரு

- செவ்வேள்

- காயாம்பூ வண்ணன்

- நீனிற நெடியோன்

- வால் வளை புரையு மேனி

எனத் தெய்வங்களுக்கொல்லாம் பல வர்ணங்களைச் சாட்டி வர்ணித்திருந்தமையினூடாக அதனை உணர்ந்து கொள்ள முடியும். சனிக்கோளை கரியவன் எனவும், காளியை கரிய கண்டத்தவள் என்றும் குறிப்பிட்டுள்ளமையும் நோக்கத்தக்கது. பவள வாய், தவள நகை, முல்லைப் பண், குவளைக் கண், கார்கூந்தல், மாமேனி என்பனவும் உறுப்புக்களை வண்ணத்துடன் தொடர்புபடுத்திப் பாடிய பாடற் சொற்களாகவே காணப்படுகின்றன. நீலத்தைக் கருப்போடு வேறுபாடின்றி எண்ணுகின்ற தன்மையும் வண்ணத்தின் சிந்தனைக்குத் தக்க சான்றாகும்.

'கோபத் தன்ன தோயாப் பூந்துகில்

விளக்கழ லுருவின் விசியுறு பச்சை

குருதிப் பூவின் குலைக்காந்தட்டே'

என்ற பாடலினூடாக அதனை அறிந்து கொள்ள முடியும். இவ் வண்ணங்களின் அறிவே ஓவியர்களுக்குப் பயன்பட்டிருக்க வேண்டும். நிறமூட்டுதல், தோய்த்தல் என்பன அக்கால ஆடைத் தொழிலாளர்களுக்கும் உரிய செயற்பாடாக இருந்துள்ளது. என்பதுவும் குறிப்பிடத்தக்கது.

\section{ஆலயங்களிலும், மக்கள் வாழ்வியலிலும் காணப்பட்டதான ஓவியங்கள் பற்றிய சிந்தனைகள்}

சுதைகளினால் அழகுபடுத்தப்பட்ட கோயில்களின் மாடங்களில் பல்வேறுபட்ட செயற்பாடுகளைக் குறிக்கும் ஓவியங்கள் வரையப்பட்டிருந்தன என்று பட்டினப்பாலை குறிப்பிடுகின்றது. கோட்டோவியம். சிற்போவியம், வண்ணோவியம், சுவரோவியம் எனும் பல வகைப்பட்ட ஓவிய வகைகள் ஆலயங்களை அழகுபடுத்தியுள்ளன. வானகக் காட்சி, வையகக் காட்சி என்று இயற்கையின் கோலங்களனைத்தையும் 
ஓவியங்களாக வரைந்திருந்தனர். தமக்கு எக்காட்சியின் நினைவு அவசியமாகின்றதோ அக் காட்சியின் நிழலை ஓவியமாக்கி நினைவுகூர்ந்து இரசித்தனர் (Padmanabhan,2011).

'மதிசேர்ந்த மகவெண்மீன்

உருக்கெழுதிற லுயர் கோட்டத்து

முருகமர்பூ முரண்கிடக்கை

வரியணிசுடர் வான்பொய்கை

இருகாமத் திணையேரி'

என்ற பாடலில் கூறப்படுவதான ஏரிக்கரைக் காட்சியானது ஓவியமாக வரையப்பட்டிருக்க வேண்டும் என்று பலரும் கருத்துத் தெரிவிக்கின்றனர். ஓவிய அழகுக் கலையானது இல்லங்களிலும், மாடங்களிலும், அரண்மனைகளிலும், கோயில்களிலும் மக்கள் கூடும் பொது இடங்களிலும் வரையப்பட்டிருந்தன. பூவும், கொடியும், பறவையும், விலங்கும், பாவையும், தெய்வமும், எனப்பல உருவங்களை எழுதி வரைந்து அவ்விடங்களையெல்லாம் அழகுபடுத்தியிருந்தனர். சிறுவர்களுக்குரிய விளையாட்டுப் பொருட்களிலும் ஓவியம் முக்கிய இடம்பிடித்திருந்தது.

'பெருமுது செல்வர் பொன்னுடைப் புதல்வர்

சிறுதோட் கோத்து செவ்வரிப் பறையின்

கண்ணகத் தெழுதிய குரீஇப் போலக்

கோல்கொண் டலைப்பப் புடிஇயர் மாதே'

எனத் தலைவனைக் கொண்டு செல்லும் குதிரைகள் சிறுவர் தோட்கண் பிணித்த பறையின் கண் எழுதிய குருவி போல அடிபடும் என்று தோழி கூறவதாக முதுகூற்றனார் மொழிகிறார். இப்பாடல் பெரும்பாணாற்றுப்படைப் பாடலாகும். பண்டைத் தமிழ் மக்கள் ஓவியம் வரையப்படும் கருவியை துகிலிகை என்றழைப்பதாகவும் அவர் குறிப்பிட்டுள்ளார்.

'ஓவ மாக்கள் ஓள்ளரக் கூட்டிய துகிலிகை'

என்ற வரிகளினூடாக அதனை உணர்ந்த கொள்ளலாம். நற்றிணைப் பாடலில் சுந்தரத்தனார் மடன்மா ஏறும் தலைவன் தலைமகளை ஓவியமாகத் தீட்டி ஊர்க்கண் பலரும் அறியுமாறு நிகழ்த்தும் பெருந்திணைச் செயலைப் பற்றிப் பாடியுள்ளார். இதன் வாயிலாக ஓவியக்கலை பண்டைத் தமிழர்களின் வாழ்வியலோடு இயைந்துள்ளது என்பது அறியக் கிடைக்கின்றது. இப்பாடல் வரியின் படி ஓவியனை 'கிளவி வல்லோன்' என அழைத்திருந்தமையும் தெளிவாகின்றது.

'கிளவி வல்லோன் எழுதியன்ன காண்டகு வனப்பின் ஐயள்'

என்ற வரிகளின் வாயிலாக தான் காணும் காட்சியை கண்டது போலவே அவ்வாறாகவே வரையும் ஓவியர்கள் அக்காலத்திலிருந்துள்ளமை தெரியவருகின்றது.

'வினையிடை விலங்கல் போலும் புனைசுவர்ப்

பாவை யன்ன பழிதீர் காட்சி'

தோழியானவள் தன் தலைமகளின் பழிதீர்க் காட்சிக்கு ஒ ஒப்பான பேரழகைச் சுவரோவியம் அனையது என்பதாக மேற்கண்ட பாடல் நற்றிணையில் அம்மெய்யன் நாகனாரால் மொழியப்பட்டுள்ளதைக் காணலாம். சுவரோவியமாகப் பெண்ணொருத்தி வரையப்பட்டிருந்ததனை இப்பாடல் வரி சுட்டுகின்றது. இவற்றுடன் கொல்லிமலையில் வரையப்பட்ட பாவை ஒன்றினைப் பற்றிய செய்தியைப் பரணர் குறுந்தொகையில் குறிப்பிடுகின்றார். இதனை ஓவியம் என்றும் சிற்பம் என்றும் கருதுகின்றனர். ஓவியர்கள் வல்லவர்களாகவும் வர்ணிக்கப்பட்டு அழைக்கப்பட்டிருந்தனர். வல்லோன் எனப்படும் ஓவியனால் வரையப்படும் காட்சியானது மனதிலே என்றும் பதிந்திருக்க வல்லது என்பதை பரிபாடலொன்று குறிப்பிடுகின்றது. 
'பல்லூழ் இவையிவை நினைப்பின் வல்லோன்

ஓவத் தெழுதெழில் போலும் மாதடிந்து

அட்டோய் நின் குன்றின் மிசை'

என்றவாறு அப்பாடல் வரிகள் அமைந்து காணப்படுகின்றன. ஓவியம் கருத்துப் பரப்ப நற் கலைக்கருவியாகும். கற்காத பாமரர்களும் கல்வி கற்றவர்களும் ஒருங்கே ஈடுபடும் கலைவடிவம் ஓவியமாகும். செம் பஞ்சுக் குழம்பும், குங்குமக் குழம்பும், கொண்டு நகம், நெற்றி, மார்பு ஆகியவற்றில் பூசிக்கொள்ளும், வரிக் கோடுகளாய் வரைந்து கொள்ளும் மகளிர் ஒப்பனை வழக்கங்களும் ஓவியத்தின் வழியாகவே பெறப்பட்டன. என்று பரிபாடல் குறிப்பிடுகின்றது. எனவே ஓவியம் வரைதற்கு பல்வேறு ஊடகங்கள் இருந்தாலும் கூட அழகிய மகளிர் தங்கள் உடலையே ஊடகமாக்கி அழகு கூட்டியுள்ளனர் என்றென்ணும்போது ஓவியம் தானும் அழகாயிருந்து தான் சேருமிடத்தினையும் அழகாக்க வல்லது என்பது தெளிவாகின்றது.

'ஆயிடை மாயிதழ் கொண்டோர் மடமாதர் நோக்கினாள்

வேயெழில் வென்று வெறுத்ததோள் நோக்கிச்

சாயிழை பிண்டித் தளிர் காதிற் தையினாள்

பாய்குழை நீலம் பகலாகத் தையினாள்

குவளை குழைக்காதின் கோலச் செவியின்

இவள் செரீஇ நான்கு விழிபடைத்தாளென்று

நெற்றி விழியா நிறைத்திலகமிட்டாளே

கொற்றவை கோலங்கொண்டதோர் பெண்'

எனும் பரிபாடல் பெண்கள் தம்மை ஓவியங்களாலும் வண்ணங்களாலும் அழகு செய்தனர் என்பது பற்றிக் கூறிச் செல்கின்றது. அத்துடன் அக்காலப் பெண்கள் மார்பில் 'தொய்யில்' (சந்தனம், குங்குமம், தேவதாரம், அகில் என்பன சேர்ந்த கலவை) எழுதுதலைப் பற்றிக் கலித்தொகை குறிப்பிடுகின்றது.

'என்தோள் எழுதிய தொய்யிலும் யாழநின்

மைந்துடை மார்பில் சுணங்கும் நினைத்துக்காண்'

தெய்யில் என்பது நீர்க்கொடி போன்று கோட்டோவியமாகிய ஒப்பனையாகும் என்று அனந்தராமையர் தன்னுடைய கலித்தொகைப் பதிப்பிலே குறிப்பிடுகின்றார். ஆறுபடை வீடுகளிலொன்றான திருப்பரங்குன்றத்தில் அமைந்த எழுத்துநிலை மண்டபம் ஓவியங்களினால் நிரம்பப்பெற்றதாகும். அகலிகை பெற்ற சாபம் குறித்ததான ஓவியங்கள் இங்கே காணப்படுவதாக நப்பண்ணனார் குறிப்பிடுகின்றார்.

'இரதி காமன் இவளிவன் எனாஅ

விரகியர் வினவ வினாவிறுப் போரும்

இந்திரன் பூசை இவள் அகலிகையிவன்

சென்ற கவுதமன் சினனுறக் கல்லுரு

ஒன்றிய படியிதென்றுரைசெய் வோரும்

இன்ன பலபல எழுத்துநிலை மண்டபம்' 
என்ற பாடல் வரிகளினூடாக அவரது கூற்று மெய்ப்படுகின்றது (Sithambaranar, 2004). எனவே வடமொழிப் புராணக் கதைகளின் செலவாக்கு ஓவியக்கலையின் வாயிலாக வெளிக்காட்டப்படுகின்றமையும் புலனாகின்றது. வண்ணம் ஒளிவீசும் படி தீட்டப்படுவதனை,

'வண்ணம் தெளிர

என்று பரிபாடல் குறிப்பிடுகின்றது. ஓர் ஓவியன் அழிந்தாலும் அவன் வரைந்த ஓவியம் காலம் காலமாய் நிலைத்து நின்று அவனை நினைவுபடுத்த வல்லது. 'தாயுயிர்செய்த பாவை போல......' எனும் பாடல் வரி அதனை உணர்த்தும். ஆகவே ஒருவர் காணும் காட்சியானது அவர் உள்ளத்திலே என்றும் பதிந்து நிற்கும் தன்மை வாய்ந்தது என்பது மறுப்பதற்கில்லை.

'முன்னங்காட்டி முகத்தினுரையா

ஓவச் செய்தியின் ஓன்று நினைந்தொற்றி'

எனும் அகநானூற்றும் பாடல் அகத்தின் அழகு முகத்தில் தெரியும் எனும் பழமொழியை நினைவுபடுத்துவதாயுள்ளது. அதாவது ஓவியம் போல முகமும் உணர்ச்சியை தெளிவுற எடுத்துக்காட்ட வல்லது. உளக்குறிப்பை உணர்த வல்லது ஓவியம் என்பதே இப்பாடலின் கருத்தாகும்.

இவ்வாறாக சங்கப் பாடல்களைப் போன்று சங்கமருவிய காலப் பாடல்களிலும் ஓவியம் பற்றிய வர்ணனைகள் இடம்பெறுவதனைக் காணலாம்.

'ஓருமுக எழினியும் பொருமுக எழினியும்

கரந்துவரல் எழினியும் புரிந்துடன் வகுத்தாங்கு

ஓவிய விதானத்து உரைபெறு நித்திலத்து

மாலைத் தாமம் வளையுடனாற்றி'

என கடலாடு காதை கூறுகின்றது. அதாவது முத்துமாலைகள் மேலமைந்த ஓவிய விதானத்தினின்று கோவலனும். மாதவியும் யாழிசைத்தனர். என்பதனை மேற்கண்ட வரிகள் உணர்த்துகின்றன. இங்கே ஓவிய விதானம் பற்றிய குறிப்பு இடம்பெறுவதனைக் காணலாம்.

நல்லார் உரையில் கோசிகம், பீதகம், பச்சிலை, அரத்தம், நுண்டுகில், சுண்ணம், வடகம், பஞ்சு, இரட்டு, பாடகம், கோங்கலர், காத்தூலம், வெண்பொத்தி, செம்பொத்தி. பணிப்பொத்தி எனப்பல ஆடை வகைள் பண்டைத் தமிழ்களின் கலாசாராத்திலே காணப்பட்டன எனும் கூற்றுள்ளது. இவ் ஆடைகளிலெல்லாம். ஓவிய வேலைப்பாடுகளும், வண்ணவேலைகளும், காணப்பட்டிருந்தன. இவ் வேலைப்பாட்டினைச் 'சித்திரக் கரணம்' என அரங்கேற்றுக் காதை கூறுகின்றது. ஓவியர்களை 'கண்ணுள்வினைஞர்' என்று இந்திரவிழாவூரெடுத்த காதை கூறுகின்றது. சித்திரச் சூடகம் என்று கடலொடு காதையில் சூடகவணி சித்திரத்தொழிற் சிறப்புப் பெற்றமை பற்றிக் கூறுகின்றது.

வண்ணமும், சுண்ணமும் அரக்கும், மெழுகும், பூச்சும் கொண்டியற்றும், ஒருசார் சிற்பவினையாகிய கம்மியத் தொழிலும் ஓவியம் என்றே கருதப்பட்டிருந்தது. யாழின் உறையில் ஓவியம் தீட்டுதல் பண்டைய வழக்கமாகும். 'சித்திரப் படம்' என்று இவ் உறையை கானல்வரி குறிப்பிடுகின்றது. அரண்மனையில் 'சித்திர மாடம்', சித்திர மண்டபம் என்பன ஓவியப் பொலிவுடையனவாய் அமைந்துள்ளன.

'செம்பியன் மூதூர்ச் சென்று புக்காங்கு

வச்சிரமவந்தி மகதமொடு குழிஇய

சித்திர மண்டபத்திருக்க'

எனச் செங்குட்டுவன் சித்திர மண்டபத்திலிருந்தமை பற்றிய செய்தி வெளித் தோற்றுகின்றது. அரசிகள் வாழும் அந்தப்புரத்திலும் அரசி துயிலுறும் கட்டிலின் மேலும் ஓவிய விதனம் அமையுமென நீர்ப்படைக்காதை குறிப்பிடுகின்றது. 
'முத்துநிரை கொடி தொடர் முழுவதும் வளைஇய

சித்திர விதானத்துச் செய்பூங் கைவினை

மடையமை செலவின் வான்பொற் கட்டில்'

என்று அப்பாடல் வரிகள் அமைந்து காணப்படுகின்றன. 'பெருஞ்சித்திரத்தன்', 'சித்திரத்தன்' எனும் பெயர்களும் வழக்கிலிருந்தன. மாதவியின் தாயின் பெயர் 'சித்திராபதி' என்பதும் நினைவுகூரத்தக்கது. அழகிய பெண்களை ஓவியமாக வரைந்து பொதுவிடங்களில் பேணல் என்பதும் வழக்கமாயிருந்தது. என்று மணிமேகலை கூறுகின்றது. மணிமேகலையை உதயகுமாரன் ஓவியப்பாவை என எண்ணிய செயலைச் செயலைச் சாத்தனார் குறிப்பிடுகின்றார்.

'ஓவியன் உள்ளத் துள்ளியது வியப்போன்

காவியங் கண்ணி யாடுதல் தெரிந்து

மேவிய பளிங்கின் விருந்திற் பாவையிது

ஓவியச் செய்தியென் றொழிவேன் முன்னர்'

எனும் பாடல் மூலம் உண்மைத் தோற்றமென்றே கருதும் படியான தத்ரூபமான ஓவியம் வரையப்பட்டதான செய்தி புலனாகின்றது. அதனாலேயே உதயகுமாரன் குழப்பத்திற்கு ஆழானமையும் குறிப்பிடப்படுகின்றது. மலும் சிலப்பதிகாரத்திலே கூறப்படும் 'சித்திரசேனன்' எனும் ஓவிய வல்லோனாகிய கந்தர்வனின் நண்பன் துவதிகன் என்பவன் கந்திற் பாவையில் நிலைபெற்றுறையும் செய்தி மணிமேகலையிலும் குறிப்பிடப்பட்டுள்ளது.

'நாடக மகளிர்க்கு நன்கனம் வகுத்த

ஓவியச் செந்நூல் உரைநூற் கிடக்கையும்'

எனும் வரிகள் ஆடல் மங்கையர்களுக்கு ஓவியக் கலையும் இன்றியமையாதது என்று பாடம் சொல்கின்றது. மணிமேகலைக்குப் பின் தமிழக ஆட்சி களப்பினர் வயப்பட்டமையினால் தமிழ் சார்ந்த இலக்கிய வளர்ச்சி குன்றியது. களப்பினர்களுக்குப் பின் பல்லவர் காலத்திலேயே ஓர் சீரான கலையெழுச்சியும், மொழியெழுச்சியும் ஏற்பட்டது. எனினும் சங்ககால கலைத் தன்மைக்கும் பல்லவர் கால கலைத் தன்மைக்கும் இடையே குறிப்பிடத்தக்க வேறுபாடுகள் காணப்படுகின்றன. கலை முழுவதும் சமயம் சார ஒருசேர திரும்பிய காலமாக பல்லவர் காலம் காணப்படுகின்றது. ஓவியத்தைப் பழைய இலக்கியங்கள் 'படம்' என்கின்றன. 'படாம்' என்பது சித்திரம் எழுதப்பட்ட சித்திரச் சீலையை குறிக்கும் என்று மயிலை சீனி வேங்கடசாமி குறிப்பிடுகின்றார்.

'மடத்தகை மாமயில் பனிக்குமென் றருளிப்

படாஅம் ஈத்த கெடாஅ நல்லிசைக்

கடாஅ யானைக் கலிமான் பேக'

எனும் பரணரின் பாடல் அதனைக் குறிப்பிடும். இவ்வாறாக பண்டைத் தமிழர்களிடம் காணப்பட்ட ஓவியக் கலை பற்றிய சிந்தனைகளை அறிய முடியும். சித்திரத்தை மனிதன் வரையத் துவங்கியதிலிருந்தே படிப்படியாக தான் வரைந்த ஓவியத்தை உருவமாக்கிக் காண ஆசைகூர்ந்தான். அவ்வாறு தோன்றியதே சிற்பக்கலையாகும். இச் சிற்பக் கலைகுறித்த சிந்தனைகள் பண்டைத் தமிழர்களிடம் எவ்வாறிருந்தன என்பது பற்றிப் பின்வருமாறு ஆராய்வோம்.

\section{சங்க மற்றும் அறநெறிக்கால நூல்களில் சிற்பம் பற்றிய குறிப்புக்கள்.}

சிற்பம் என்ற சொல் சங்க நூல்கள் எங்கிலுமே காணக்கிடைக்கவில்லை. எனினும் சில், சிலை சிலம்பு, என ஓசைப் பொருண்மை பயக்கும் சொற்கள் வழக்கதிலிருந்துள்ளன. சிலை என்ற சொல் சங்க நூல்கள் பலவற்றில் காணப்படுகின்றன. எனினும் அச் சொல் வில்லினைக் குறிக்கவே பயன்பட்டுள்ளது 
என்பது இலக்கிய ஆய்வாளர்களின் கருத்தாகும். நாம் இன்று காணும் தென்முகக் கடவுள் பற்றிய சிந்தனைகள் சங்ககாலத்திலும் காணப்பட்டுள்ளது. அதர்வண மகரிசியின் அதர்வண வேதத்தினை அங்கீகரிக்க சிவபெருமான் எடுத்த வடிவம் தட்சணாமூர்த்தி வடிவம் என்று சிவபுராணம் குறிப்பிடுகின்றது. இவரையே சங்க நூல்களிலொன்றான, கலித்தொகை பின்வருமாறு குறிப்பிடுகின்றது.

'மாமலர் முண்டகந் தில்லையோ டொருங்குடன்

கான லணிந்த உயர்மணல் எக்கர் மேல்

சீர்மிகு சிறப்பினோன் மரமுதல் கைசேர்த்த

நீர்மலி கரகம்போல் பழந்தூங்கு முடத்தாழை

பூமரல்ந் தவைபோலப் புள்ளளல்குந் துறைவகேள்'

எனவரும் வரிகள் குடைவரையாக அமைத்த கழுகுமலைக் கோயிலிற் காணப்பெறும் தட்சணாமூர்த்தி வடிவத்தினைச் சிறப்பிக்கின்றன. தட்சணாமூர்த்தி சிற்பம் சிற்பக் கலை வளர்ந்த நிலையில் தோற்றம்பெற்றதாக அறியமுடிகின்றது. ஆயினும் அதற்கு பலநூறு ஆண்டுகளுக்கு முன்னரே பண்டைத் தமிழகத்தில் சிற்பக் கலை இருந்துள்ளமைக்கு அங்கு கண்டெடுக்கப்பட்ட நடுகற்களே சான்றாக அமைகின்றன.

'காட்சி கால்கோள் நீர்ப்படை நடுகல்

சீர்தகு மரபில் பெரும்படை வாழ்த்தலென்று

இருமூன்று மரபிற் கல்லொடு புணரச்

சொல்லப் பட்ட எழுமூன்று துறைத்தே'

என்ற தொல்காப்பிய வரிகள் உயிரிழந்த மதலைக்குக் கல்லெடுத்து நினைவுகூர்ந்ததைக் குறிப்பிடுகின்றது. நிரைப்போரின் இறுதியில் இடம்பெற்ற இவ் ஈமச் சடங்கு பற்றியே இப்பாடல் குறிப்பிடுகின்றது.

'பெயரும் பீடு மெழுதி அதர்தொறும்

பீடிய சூட்டிய பிறங்குநிலை நடுகல்'

எனும் வரிகள் கல்லொன்றிலே இறந்தோர் பெயரும் பெருமையும், எழுதப்படும் மரபு இருந்ததாகக் காட்டுகின்றது. எனவே இவ்வாறாகவே நடுகல் வழிபாட்டின் வாயிலாக சிற்பம் அமைக்கும் முறை பண்டைத்தமிழகத்தில் உருவாகியிருத்தல் வேண்டும் எனக் கருதப்படுகின்றது.

\section{சங்க இலக்கியங்களில் பாவை, மற்றும் பாவையின் பழமை பற்றிய குறிப்புக்கள்}

சங்க காலத்தில் வார்த்தல், செதுக்கல், புனைதல் என்ற தொழில் வினைகளினூடாக உருவங்கள் செய்யப்பட்டன. அக்காலப் பெண்கள் மணலில் மண்பாவை செய்து விளையாடும் வழக்கத்தினையும் கொண்டிருந்தனர். பேதைப் பருவத் தலைமகள் தான் செய்த வண்டற் பாவையைத் திரையலைத்ததெனக் கேட்டு கடல் மீீு கடும் கோபம் கொள்வதான வர்ணனைகள் சங்க இலக்கியங்களிலே இடம்பெற்றுள்ளன.

'இதுஎன் பாவைக்கு இனியநன் பாவை

இதுஎன் பைங்கிளி எடுத்த பைங்கிளி

இதுஎன் பூவைக்கு இனியசொற் பூவைஎன்று

அலமரு நோக்கின் நலம்வரு சுடர்நுதல்' 
என்று பொம்மைப் பாவைகளைக் கொண்டு இளையாரைத் தேற்றுமாறு கூறப்பட்டதான பாடல் வரிகளும் பண்டைத் தமிழர்களின் சிற்பத்திறனை வெளிக்காட்டுகின்றன. விளையாட்டுப் பாவையான சிறு உருவிலிருந்து மனித முழு உருவம் வரையான சிற்பங்கள் பற்றிய செய்திகள் பல சங்க இலக்கியங்களில் குறிப்பிடப்பட்டுள்ளன. குறுந்தொகையிலே பாவை ஒன்றுடன் தொடர்பான கதையொன்று கூறப்படுகின்றது. அதாவது, அரசர்குரிய மாமரம் ஒன்று கடுங்காவலின் கீழே யாரும் அவ் மரத்தை நெருங்காதபடி பாதுகாக்கப்பட்டு வந்தது இருப்பினும் அதன் தீஞ்சுவை கொண்ட மாங்கனியொன்று நதியிலே விழுந்து ஓடிச்சென்று ஓர் பெண்ணின் கைகளைச் சேர்கின்றது. அவளும் அதையறியாது உண்டு விடுகின்றாள். அத்தவறுக்குத் தண்டனை கிடைக்கும் எனவஞ்சிய பெண்ணின் பெற்றோர் தம் மகளைப்போன்ற பொன் பாவையொன்றைச் செய்து, அத்துடன் 81 களிற்று யானைகளையும் அபராதமாக தண்டப்பொருளாக நேர்ந்தனர். எனினும் மன்னன் அவற்றை ஏற்காது அப்பெண்ணைக் கொன்றுவிடுவதாக அக் கதை அமைகின்றது. இதன்படி ஓர் உருவத்தினைப்போல உலோகப் பதுமைகள் செய்யும் வழக்கம் சங்ககாலத்தில் இருந்துள்ளது என்று அறியலாம்.

'அவள் நிறை பொன் செய் பாவை'

என்ற வரிகள் அப்பெண் பாவையைச் சுட்டுவதாகவுள்ளன. அதேபோல அகநானூற்றுப் பாடல் ஒன்று,

'வளங்கெழு முசிறி ஆர்ப்பெழ வளை,

ஆருஞ்சமங் கடந்து படிமம் வவ்விய

நெடுநல் யானை அடுபோர்ச் செழியன்'

என்று செழியன் என்பவனது போர்த்திறம் பற்றிக் குறிப்பிடுகின்றது. இ இங்கு செல்வர் மனைகளிலும், அரசர் வாழிடங்களிலும், அழகும் உடமைப் பெருக்கும் பொலியப் பொற்பாவை செய்து வைத்திருந்தனர் என்று கூறப்படுகின்றது. எனவே அழகுக்காக படிமங்கள் செய்து வாழிடத்தை அலங்கரிக்கும் வழக்கம் பண்டையத் தமிழகத்தில் நிலவியுள்ளது என அறியலாம். உறுப்பழகு மிக்க சிற்பத் தொழில்மிகுந்தனவாக இப்பாவைகள் காணப்படுகின்றன. படிமம் எனும் சொல் தெய்வச் சிற்பங்களையும் குறிக்கப் பயன்பட்டுள்ளதாக இலக்கியச் சான்றுகள் கிடைக்கப்பெறுகின்றன.

'கைவினை முற்றிய தெய்வப் படிமத்து வித்தக ரியற்றிய விளங்கிய

கோலத்து'

எனக் கண்ணகியின் தெய்வப் படிமம் சிலப்பதிகாரத்திலே (28: 221: 29) சிறப்பிக்கப்படுகின்றதனை அதற்கு உதாரணமாகக் கூறலாம். ஆற்றல் மிக்க கடவுளர்களைக் காட்ட கைகளும், தோளும் பலவாகப் படைக்கப்பட்டன. தொல்காப்பிய நீர்படைத்துறைக்கும், சிலப்பதிகார நீர்படைத்துறைக்கும் இடையே கால இடைவெளி அதாவது சங்க மற்றும் சங்கமருவிய காலத்திற்கு இடையேயான கால இடைவெளி சிற்பக் கலை வளர்ச்சியிலும் மாறுபாடுகளைத் தோற்றுவித்தது. சங்க காலத்து மக்களிடம் உருஅமைத்து அவ்உருவை வழிபடும் வழக்கம் மிகக் குறைவாகவே இருந்துவந்துள்ளது. ஆனால் பின் தோன்றிய சங்கமருவிய காலத்தில் மிக அழகான சிற்பங்கள் படைக்கப்பட்டுள்ளன. கண்ணகிக்கு அழகான சிற்ப்பம் படைக்கப்பட்டது பற்றி சிலப்பதிகாரம் பின்வருமாறு விவரணமாகக் குறிப்பிடுகின்றது.

'நன்னா டணைந்து நளிர்சினை வேங்கைப்

பொன்னணி புதுநிழற் பொருந்திய நங்கையை

அறக்களத் தந்தண ராசாண் பெருங்கணி

சிறப்புடைக் கம்மியர் தம்மொடுஞ் சென்று

மேலோர் விழையு நூனெறி மாக்கள்

பால்பெற வகுத்த பத்தினிக் கோட்டத்து

இமையவ ருறையு மிவைச் செவ்வரைச் 
சிமையச் சென்னித் தெய்வம் பரசிக்

கைவினை முற்றிய தெய்வப் படிமத்து

வித்தக ரியற்றிய விளங்கிய கோலத்து'

இவ்வாறு அமைந்த வரிகள் கம்மியரான் இயற்றிய கைவினை முற்றிய தெய்வப் படிமம் என்கின்றது. ஆகவே தெய்வப் படிமங்களை வடிப்பதற்கு கம்மியன் எனப்பட்ட தொழிலாளர்கள், கைத்திறமையாளர்கள் காணப்பட்டுள்ளனர் என்பதும் தெய்வப் படிமங்கள் சங்கமருவிய காலத்தில் சிறப்புற அமைக்கப்பட்டு வழிபாடுகள் இயற்றப்பட்டுள்ளன என்பதும் தெளிவாகின்றது. சங்கமருவிய காலத்திலிருந்து ஏறத்தாழ 600 ஆண்டுகள் பின்தள்ளியே தெய்வ உருவங்கள் பற்றிய செய்திகள் மிகுதியாகக் காணக்கிடைக்கின்றன. எனினும் சங்ககால முற்பகுதியில் படிமங்கள் எதுவும் கிடைக்கவில்லை என்று கூறிவிட முடியாது.

'களிறுகெழு தானைப் பொறையன் கொல்லி

ஒளிறு நீர் அடுக்கத்து வியலகம் பொற்பக்

கடவுள் எழுதிய பாவையின்

மடவது மாண்ட மாஅ யோளே'

என்ற பரணரின் அகப்பாடற் பகுதி சங்க காலத்து முற்பகுதிக்கு உரியதான கொல்லிப் பாவையின் படிமம் குறித்த செய்தியை எடுத்துரைக்கின்றது. இது ஓவியமாகவும் கருதுவோர்கள் உள்ளனர்.

'பெரும்பூட் பொறையன் பேஎழுதிர் கொல்லிக்

கருங்கட் டெய்வங் குடவரை யெழுதிய

நல்லியற் பாவை யன்னவிம்

மெல்லியற் குறுமகள் பாடினள் வரினே'

எனக் கொல்லிமலையின் மேற்கே அமைந்த பாவைப் படிமம் பற்றிப் பரணர் குறிப்பிடுகின்றார். காண்பவர் நெஞ்சம் கலங்கி மயங்கி வீழ்ந்து உயிர்விடச் செய்வதாக இப்படிமம் காணப்படுவதாக வர்ணிக்கின்றார். சிரித்தே கொல்லும் இயல்பும் இதற்குள்ளதாகவும் குறிப்பிடுகின்றார். முள்ளூர் மன்னன் கழல்தொடிக்காரி ஓரியைக் கொன்று கொல்லி மலையைச் சேர்கிறான். அங்கு 'நிலைபெறு கடவுள் ஆக்கிய பார்புகழ் பாவை' இருந்ததெனக் கல்லாடனர் குறிப்பிடுகின்றார். இதன்படி கொல்லிமலையில் வனப்புமிக்க கடவுட்பாவை அமைந்திருந்ததனை அறியலாம். அத்துடன் தெய்வத்தாற் செய்த கொல்லிப் பாவை என நச்சினார்கினியார் ஓர் உருவம் பற்றிக் குறிப்பிடுகின்றார். இவர் குறிப்பிடும் சிற்பம் கிழக்கு நோக்கி அமைக்கப்பட்டதாக இருத்தல் வேண்டும். காலை இளவெயிலை இச்சிற்பம் வாங்குமாறு அமைந்திருத்தல் கூடும். என அறியக்கிடைக்கின்றது.

'செவ்வேர்ப் பலவின் பயங்கெழு கொல்லித்

தெய்வம் காக்கும் தீதுதீர் நெடுங்கோட்டு

அவ்வெள் ளருவிக் குடைவரை யகத்து

கால்பொரு திடிப்பினும் கதழுறை கடுகினும்

உருமுடன் நெறியினும் ஊறு பலதோன்றினும்

பெருநிலங் கிளரினும் திருநல உருவின்

மாயா இயற்க்கைப் பாவை'

எனும் பரணரின் பாடல் வரிகளும் அதனை விளக்குகின்றன. மேலும் இ இச்சிற்பம் பற்றிக் கூறவிழைந்த பரணர், இச் சிற்பப் படிமமானது பல இயற்கை ஊறுகளைத் தாண்டியும் காலம் கடந்து 
நிலைபெற்றிருந்ததாகவும், நெடியதாக இருந்ததாகவும், பூதன் என்பவனால் செய்யப்பட்டதாகவும் குறிப்பிடுகின்றார். இப் படிமத்தின் சிறப்பினாலேயே பரணர் தன்னுடைய பாடல்களில் பலவிடங்களில் இப் படிமத்தையே எடுத்துக்காட்டாகக் குறிப்பிடுகின்றார்.

பழந்தமிழ்ப் பனுவல்களில் ஒன்றான பட்டினப்பாலை கடவுளின் உருவங்கள் ஓவியங்களாகவும் படிமங்களாகவும் கோயில்களில் அமைக்கப்பட்டிருந்ததாக எடுத்துக் காட்டுகின்றது. நெய்தல் நில மணற் பரப்பில் சினைச் சுறாவின் கொம்பினை நட்டு அதனில் தெய்வத்தைக் கண்டு வழிபடும் வழக்கம் பண்டைத் தமிழ் மக்களிடம் காணப்பட்ட மரபாகும். இவற்றிற்கெல்லாம் பின்பு தோன்றிய திருமுருகாற்றுப்படையானது, முருகப் பெருமானின் ஆறுபடை வீடுகள் பற்றியும், அங்கிருந்த ஓவிய சிற்பங்கள் பற்றியும் குறிப்பிடுகின்றது. இதன் பின்னர் சங்க மருவிய காலத்தில் தோன்றிய சிலப்பதிகாரத்திலே,

'பிறவா யாக்கைப் பெரியோன் கோயிலும்

அறுமுகச் செவ்வேள் அணிதிகழ் கோயிலும்

வால்வளை மேனியோன் வாலியோன் கோயிலும்

நீலமேனி நெடியோன் கோயிலும்

மாலை வெண்குடை மன்னவன் கோயிலும்

என்று பல்வேறு கடவுளர்களுக்குரிய கோட்டங்கள் அமையப்பெற்றிருந்தமை பற்றிக் குறிப்பிடுகின்றது (Swaminatha Iyer, 1920). அல்லி மலரொன்றில் திருமகள் வீற்றிருக்கவும் இருபுறம் யானைகள் பூவும் நீரும் சொரியும் காட்சி கலித்தொகைப் பாடல்களில் கூறப்பட்டுள்ளது. ஆகவே சங்ககாலத்தில் படிமக்கலை சிறப்புடனேயே காணப்பட்டுள்ளது என்பது தெளிவு. பிற்காலத்தில் தோன்றிய படிமக் கலையானது வார்ப்புக் கலையாகும். ஐம்பொன்னால் வார்ப்புச் செய்து பின்னர் உளி கொண்டு செதுக்கப்பட்டவைகளாக பிற்காலப் படிமங்கள் காணப்படுகின்றன.

\section{பிற படிமங்கள் பற்றிய குறிப்புக்கள்}

மண்ணாலும், கல்லாலும், மெழுகாலும், யாானைக் கோட்டாலும், சுண்ணத்தாலும், கண்ட சருக்கரையாலும், சிற்ப உருவங்கள் செய்யப்பட்டிருந்தன. திவாகர நிகண்டு குறிப்பிடுவது போல 10 மூலப்பொருட்களால் உருவங்கள் ஆக்கப்பட்டுள்ளமை தெரிய வருகின்றது. அன்னிய படையெடுப்புக்கள் தமிழகத்தின் கலைமாற்றத்திற்கும் துணைபுரிந்துள்ளன. அவ்வாறு வந்த வேற்று இனத்தவர்களான யவனர்கள் ஓதிம விளக்கையும், பாவை விளக்கையும் உருவாக்குவதற்கு முன்னோடிகளாக இருந்துள்ளனர் எனத் தெரிய வருகின்றது. காவற் பணியில் அமர்த்தப்பட்ட யவனர்கள் மேற்குறிப்பிட்ட விளக்குகளை அமைத்ததாக முல்லைப்பாட்டும், பெரும்பாணாற்றுப்படையும் நெடுநல் வாடையும் குறிப்பிடுகின்றன.

'யவன ரியற்றிய வினைமாண் பாவை

கையேந் தையக னிறையநெய் சொரிந்து

பரூஉத்திரி கொளீஇய குரூஉத்தலை நிமிரெரி'

என சோனகர்கள் (யவனர்கள்) அமைத்த பாவை விளக்குப் பற்றிய செய்தியை நெடுநல்வாடை குறிப்பிடுகின்றது. சிற்ப வேலைப்பாடுகள் நிறைந்த வெண்கலத்திலாலான பாவை விளக்குகள் அதிக அழகுடையன என்றும் அரண்மனைகளில் அலங்காரத்திற்கும் ஒளிக்காகவும் பயன்பட்டன என்றும் தெளியலாம். அத்துடன் அவர்களைத் தவிர மேலும் பலநாட்டுத் தொழிலாளர்கள் தமிழகக் கலைகளுக்குப் பங்களிப்பு வழங்கியுள்ளனர் என மணிமேகலை குறிப்பிடுகின்றது.

'மகத வினைஞரும் மராட்டக் கம்முர

மவந்திக் கொல்லரும் யவனத் தச்சரும் 
தண்டமிழ் வினைஞர் தம்மொடு கூடிக்

கொண்டினி தியற்றிய கண்கவர் செய்வினைப்

பவளத் திரள்காற பன்மணிப் போதிகைத்

தவள நித்திலத் தாமந் தாழந்த

கோணச் சந்தி மாண்வினை விதானத்துத்

துமனியம் வேய்ந்த வகைபெறு வனப்பிற

பைஞ்சேறு மெழுகாப் பசும்பொன் மண்டபம்'

என்றவாறு அயல் தேசத்தவர்களின் கலைப் பங்களிப்புப் பற்றி அறியலாம். அக்கால ஆலயங்களில் தூண்கள் நிறுத்தப்பட்டு காணப்பட்டன என்பதனை,

'நெடுநிலைக் கந்தி னிடவயின் விளங்கக்

குடவு ளெழுதிய பாவையாங் குரைக்கும்

எனவரும் மணிமேகலையின் வாளாலெறிந்த காதையின் வரிகள் குறிப்பிட்டுச் செல்கின்றன. இவற்றுடன் மரத்தினால் தச்சர்களால் செய்யப்பட்ட அரசன் அமர்ந்து வரும் தேர்களும் சிற்பவேலைப்பாடுகளுடன் கூடியனவாக அமைக்கப்பட்டிருந்தன.

'இருந்த வேந்தன் அருந்தொழில் முடித்தெனப்

புரிந்த காதலொடு பெருந்தேர் யானும்

ஏறிய தறிந்தன் றல்லது வந்தவாறு'

என மாசாத்தியார் குறிப்பிடுகின்றார். எனவே சங்ககாலத்தில் தேர் செய்யும் முறைமை காணப்பட்து என அறியலாம். தேர் செய்வதற்கான இலக்கண நூல்களும் காணப்பட்டிருந்தன.

'பல்கதி ராழி மெல்வழி யறுப்பக்

காலென மருள ஏறி நூலியல்

கண்ணோக் கொழிக்கும் பண்ணமை நெடுந்தேர்

வல்விரைந் தூர்மதி நல்வலம் பெறுந'

என்று பேயனார் தேர் செய்வதற்கான இலக்கண நூல் பற்றிக் குறிப்பிடுகின்றார். அத்துடன் கதவுகள் பலவும் சிற்ப வேலைப்பபாடுகளுடன் அமைக்கப்பட்டுள்ளன. போர்க் கதவு, அரண்மனைக்கதவு போன்ற பல கதவுகள் செதுக்கப்பட்டுள்ளன.

'எழூஉப்புறந் தரீஇப் பொன்பிணிப் பலகைக் குழூஉநிலைப்

புதவிற் கதவு'

எனக் கணைய மரத்தாலும், கரும்பொன்னாய இரும்பாலும், தெழிலமைதி பெற்ற கதவம் காணப்பட்டமை பற்றிக் குறிப்பிடப்பட்டுள்ளது. நெடுநல்வாடை கூறும் கதவமைப்பானது அக்காலச் சிற்பநூல் ஒழுங்கு பற்றிச் சிறப்பாகக் கூறுகின்றது.

'பருவிரும்பு பிணித்துச் செவ்வரக் குறீஇத்

துணைமாண் கதவம் பொருத்தி யிணைமாண்டு

நாளொடு பெயரிய கோளமை விழுமரத்துப்

போதவிழ் குவளைப் புதுப்பிடி காலமைத்துத்

தாழொடு குயின்ற போராமை புணர்ப்பிற் 
கைவல் கம்மியன முடுக்கலிற் புரைதீர்ந்து

ஐயவி யப்பிய நெய்யணி நெடுநிலை'

என்று கதவு அமைக்கப்பட்ட திறம் விளக்கப்பட்டுள்ளது. குவளை மலர்களையும், பிடி யானைகளையும் அக் கதவுகளில் சிற்பங்களாக இழைத்திருந்தனர். திருமகள் நடுவே அமர்ந்திருக்க அவரின் இருமருங்கிலும் யானைகளிரண்டு நீரும் பூவும் சொரியும் காட்சியும் செதுக்கப்பட்டிருந்தது. இதனை கலித்தொகை,

'வரி நுதல் எழில் வேழம் பூநீர் மேற் சொரிதரப்

புரிநெகிழ் தாமரை மலரங்கண் வீறெய்தித்

திருநயந் திருந்தன்ன தேங்கமழ் விறல் வெற்ப'

என்றவரிகளினூடாக விளக்குகின்றது. சில கதவுகளில் அவ் அரசர்களின் இலட்சிணைளும் செதுக்கப்பட்டன. புலிச் சின்னம் பொறித்த கதவுகளும் காணப்பட்டிருந்தன.

'ஏழெயிற் கதவம் எறிந்துகைக் கொண்டு

பேழ்வாய் பொறிக்கு மாற்றலை'

என்றும் 'விடற்புலி பொறித்த கோட்டை' என்றும் புறநானூறு கூறுகின்றது. மேலும் சிலப்பதிகாரமானது கைவினைப் பொருட்களைப் படைப்பவர்களைப் பல்வேறு பெயர்களினால் குறிப்பிடுகின்றது.

- கஞ்சகாரர்

- செம்பு செய்குநர்

- வெண்கலத்தால் மணிகளும் பாவைகளும், பாத்திரங்களும் செய்பவர்

- மரங்கொல் தச்சர்

- செம்பு உலோகம். துகடுகள் செய்பவர்.

- கருங்கைக் கொல்லர் - வேல், வாள், கோடரி, மணி, ஊசி, ஆணிகள், பட்டயங்கள் செய்தனர்.

- மண்ணீட்டாளர் - சுதையால் பாவைகளைச் செய்தனர்

- பொன்செய் கொல்லர் - பொன்னாலும், வெள்ளியாலும் சிற்பம் மிக்க அணிகள் இயற்றினர்

- நன்கலம் தருநர் - விலைமதிப்பிலா இரத்தினங்களை நகையிடை பதித்து அணிகலமியற்றினர்.

என்போர் எல்லாம் சிற்ப அறிவுத் திறன் வாய்ந்தவர்கள் ஆவர். இவர்களை 'பழுதில்செய்வினைப் பால்கெழு மாக்கள்' என்று பொதுப்பெயரினால் சிலப்பதிகாரம் சுட்டுகின்றது. சங்கினை அறுத்து அதனில் சிற்பங்களை செய்து அணிகலன்களாக அணியும் வழக்கமும் சங்காலத்தில் வழக்கிலிருந்துள்ளது. வளையல்களாக அறுக்கப்பட்ட சங்கில் நுணுக்கமான வேலைப்பாடுகள் காணப்டிருந்தன என்று அகநானூறு குறிப்பிடுகின்றது. இச்சங்கில் சிற்பம் செய்வோரை, 'கோடுபேழ் ஆடையினர்' என மதுரைக்காஞ்சி குறிப்பிடுகின்றது. 'மயன்' என்பவர் பற்றிய குறிப்புக்கள் பலவிடங்களில் வருகின்றன. இவர் தேவதச்சன் எனவும் அசுர தச்சன் எனவும் கூறப்படுகின்றது. இதிகாசங்களின் படி இலங்கேஸ்வரனான இராவணனது மனைவியான மண்டோதரியின் தந்தையே மயன் என்றும் அறியக் கிடைக்கின்றது. இவர் உலக அறிவுத் தூணொன்றில் துவதிகன் எனும் ஒருவனைப் பாவையாகச் செதுக்கியதாக மணிமேகலை குறிப்பிடுகின்றது.

'மன்பெருந் தெய்வக் கணங்களி லுள்ளேன்

துவதிக னென்பேன் தொன்று முதிர் கந்தின்

மயனெனக் கொப்பா வகுத்த பாவையின்

நீங்கேன் யானென் னிலையது கேளாய்'

எனக்குறிப்பிடும் வரிகள் மனிதனை சிலையாக உருவடிக்கும் முறைமை மணிமேகலை காலத்திலேயே தோன்றிவிட்டதனைக் காட்டுகின்றது. சிலப்பதிகாரமும், மணிமேகலையும் கூறுவதிலிருந்து மயமதம் என்ற சிற்பநூலும் வழக்கிலிருந்தமை தெரிய வருகின்றது. 
ஓர் கட்டத்தில் பொதுவாக எல்லாக் கலைகளுமே மனிதம் என்ற தோளிலிருந்து மாறி பக்தி எனும் தோளில் ஏறிக்கொள்ளும். கலையொன்று அழிவடையும் நிலையிலும் அதனைக் காக்க வல்லதாக இறைநம்பிக்கை காணப்படுகின்றது. இந்த விதி சிற்பக்கலைக்கும் ஏற்புடையதே. ஆரம்பத்தில் இயற்கைப் பொருட்களை செதுக்கிய மனிதன் பின் தன்னைப் பற்றியும், தான் சார் செயற்பாடுகள் பற்றியும் சிந்தித்து அவற்றை சிற்பமாக்கினான். பின்னர் இறையுருவங்களை வடிவமைத்தான். அதன்படியே, தொடக்கத்தில் உருவ வழிபாட்டினைப் போற்றாத சமண, பௌத்த சமயங்களும், மக்களிடையே உருவ வழிபாடு சிறப்படையத் தொடங்கியதனைக் கண்டு பின்னர் தாமும் தங்கள் ஸ்தாபகர்களுக்கு உருவம் வழங்கத் தொடங்கின. அதன் பின்னரே கௌதம புத்தர், மற்றும் மகாவீரர் ஆகியோருக்கு உருவங்கள் படைக்கப்பட்டன. மணிமேகலை புத்த பீடிகை பற்றி பல இடங்களில் குறிப்பிடுகின்றது.

'இளவள ஞாயிறு தோன்றியதென்ன

நீயோ தோன்றினை நின்னடி பணிந்தேன்

நீயே யாகிநிற் கமைந்தவிவ் வாசனம்

நாமிசை வைத்தேன் தலைமிசை கொண்டேன்'

எனப் பீடிகையை புத்ததேவனாகக் கொண்டு போற்றியிருப்பதனைக் காணலாம். தர்மச் சக்கரமும் புத்த குறியீடாக வணக்கத்திற்குரியதாகியது. இவ்வாறாக சமயத்தினையும் ஆட்கொண்டது சிற்பக் கலை எனலாம். நெடுநல் வாடையானது,

'வெள்ளியன்ன விளக்குஞ் சுதையுரீஇ

மணிகண் டன்ன மாத்திரள் திண்காழ்ச்

செம்பியன் றன்ன செய்வுறு நெடுஞ்சுவர்

உருவப் பல்பூஓருகொடி வளைஇக்

கருவொடு பெயரிய காண்பின் நல்லில்'

என்கின்றது. அதாவது அக்கால நிலை மாடங்களில் சுவர்கள் அனைத்திலும் கொடிகள் படர்ந்தது போன்ற சுதைச் சிற்பங்கள் காணப்பட்டன என்று அக்காலச் சிற்பக்கலை பற்றிக் குறிப்பிடுகின்றது நெடுவல்வாடை. இவ்வாறெல்லாம் பல பரிணாமங்களினூடாக தமிழர் வளர்த்த கலைகள் அனைத்தும் கடல்கோளினாலும். மீளமீள எழுந்த படையெடுப்புக்களினாலும் அழிந்து போனமை காலம் தந்த கண்ணீர் காவியமாகின்றது என்றால் மிகையல்ல.

\section{முடிவுரை}

தமிழர் வளர்த்த அழகுக் கலைகள் தனித்துவமானவை என்றும், அவை தமிழர் பண்பாட்டுடன் பின்னிப் பிணைந்தவை என்றும் அறியலாம். அத்துடன் அவ் அழகியற் கலைகளில் ஓவியக் கலையும், சிற்பக் கலையும் மிகவும் உன்னதம் வாய்ந்தவை என்றும். அவற்றின் அடியொற்றியதான வளர்ச்சியே பின்தோன்றிய பல்லவ, சோழ, விஜயநகர பேரரசுகள் காலத்தில் பெருவிருட்சமானது என்றும், அறியமுடிகின்றது. இயற்கையனர்த்தங்களினால் காணாதுபோனதும், காணாமலாக்கப்பட்டதுமான தமிழர் பண்பாட்டிற்கு மீதமாயுள்ள ஆதரமான இலக்கியங்கள் பண்டைத் தமிழர்களின் கலாவினோத ஈடுபாட்டினையும், பண்பாட்டினையும் உலகிற்கு எடுத்துக்காட்டுவனாய் உள்ளன என்பதும் தெளிவாகின்றது.

\section{References}

Navarathinam, K., (2006) Then Indhiya sirpa vadivangal, Kumaran Book House, Colombo, Sri Lanka. 
Padmanabhan, C., Raguparan, K., Prasandhan, Shree., (2011) Ara neariyum kalamum tamilaga panpadu marapukal, Hindu Religious Cultural Affairs, Colombo, Sri Lanka.

Pararajasingham, K., (2017) Sindhu samaveli nagarigamum tamilarum, Tamil sangam Batticaloa, Sri Lanka.

Sithambaranar, Sami., (2004) Pazhantamilar Vazhvum Valarchiyum, Manivasagar Pathippakam, Chennai, India.

Swaminatha Iyer, U, Ve., (1920) Silapathigaram Moolamum Arumpatavuraiyum, Adiyarkku nallaruraiyum, Commercial printer, Chennai, India.

Vaithilingam, S., (1996) Alagu Kalai Thiran, Chennai Pathippagam, Chennai, India.

Funding: No funding was received for conducting this study.

Conflict of Interest: The Author has no conflicts of interest to declare that they are relevant to the content of this article.

About the License:



(C) The Author 2022. The text of this article is open access and licensed under a Creative Commons Attribution 4.0 International License 